\title{
Combined use of anti-VEGF and anti-EGFR Monoclonal Antibodies with Photodynamic Therapy Suppresses Tumor Growth in an In vivo Tumor Model
}

\section{Ramaswamy Bhuvaneswari" ${ }^{*}$, Patricia Thong ${ }^{1}$, Gan Yik Yuen², Malini Olivo ${ }^{3}$ and Soo Khee Chee ${ }^{1}$}

${ }^{1}$ National Cancer Centre Singapore, Division of Medical Sciences, 11 Hospital Drive 169610, Singapore

${ }^{2}$ Natural Sciences and Science Education, National Institute of Education, Nanyang Technological University, 1 Nanyang Walk 637616, Singapore

${ }^{3}$ Singapore Bioimaging Consortium, Biomedical Sciences Institutes, 11 Biopolis Way, \#02-02 Helios, 138667, Singapore

\begin{abstract}
Photodynamic therapy (PDT) is a promising treatment option for cancer. It can be used to treat drug-resistant and inoperable tumors. However, one of the unsolved problems in PDT is tumor recurrence. The aim of this study is to suppress tumor regrowth by including angiogenesis inhibitors that target vascular endothelial growth factor (VEGF) and epidermal growth factor receptor (EGFR) pathways, to the PDT protocol. Human bladder cancer (MGH cell line) xenografts were induced in Balb/c nude mice. The animals treated with PDT received an intravenous injection of hypericin followed by irradiation after 6 hours, with a broadband light source through a 560-640 nm bandpass filter. A light dosage of $120 \mathrm{~J} / \mathrm{cm}^{2}$ and $100 \mathrm{~mW} / \mathrm{cm}^{2}$ was administered. Angiogenesis inhibitors, bevacizumab and cetuximab were administered at a dose of $10 \mathrm{mg} / \mathrm{kg}$. Treatment efficacy was assessed by monitoring the tumor growth inhibition of xenograft tumors. Immunohistochemistry was performed to evaluate the expression of VEGF and EGFR. Expression of the major proteins in the VEGF and EGFR pathways was investigated by immunoblotting. One-way ANOVA with Bonferroni correction was performed to analyze the data. Tumors treated with the combination of PDT and inhibitors exhibited significantly greater treatment response compared to control and PDT groups. Downregulation of VEGF and EGFR was observed in tumors treated with PDT + bevacizumab and cetuximab. Our results show that blocking VEGF or/and EGFR pathways along with PDT can effectively suppress tumor growth.
\end{abstract}

Keywords: Angiogenesis; Bevacizumab; Epidermal growth factor receptor (EGFR); Cetuximab; Photodynamic therapy (PDT); Vascular endothelial growth factor (VEGF)

\section{Introduction}

Photodynamic therapy (PDT) is a promising modality that is being used for the treatment of early cancers and to reduce tumor bulk in advanced cancers. Currently its usage is also being explored for the treatment of deep-seated tumors [1]. PDT involves the administration of a photosensitizing drug that is activated by light of a specific wavelength to selectively destroy the tumor cells. The antitumor effects result from the combination of direct tumor cell damage, destruction of tumor vasculature and/or the activation of an immune response [2]. PDT has an advantage over surgery or radiation as it can eliminate tumors selectively without causing fibrosis or scarring. However, the oxygen insult induced by PDT during treatment can trigger the expression of proangiogenic molecules as a survival mechanism, thus affecting the efficacy of the treatment $[3,4]$.

Angiogenesis, the recruitment of new blood vessels, is required for invasive tumor growth and metastasis [5]. Vascular endothelial growth factor (VEGF) plays a key role in angiogenesis, as it is a selective mitogen for endothelial cells. Epidermal growth factor receptor (EGFR) is also known to regulate angiogenesis, tumor growth and progression by upregulating VEGF [6]. Studies on preclinical models have shown that targeting both VEGF and EGFR pathways can suppress tumor growth compared to monotherapy [7-9]. A recent study performed in head and neck squamous cell carcinoma patients has demonstrated that combined targeting of EGFR and VEGF using monoclonal antibodies is well tolerated with a response rate of $16 \%$ and the disease control rate of $73 \%$ [10]. Molecular pathways that are involved directly or indirectly in tumor angiogenesis are very complex and thus interfering with a single pathway may not be sufficient to achieve optimum therapeutic efficacy. Apart from that, tumor cells also have an inherent ability to harness different growth factor signalling pathway for their growth and survival. For these reasons, developing combinatorial approaches for targeting multiple pathways without increasing the toxicity could be an attractive and effective therapeutic strategy for the treatment of cancer.

This study focuses on the use of monoclonal antibody bevacizumab and cetuximab in combination with PDT to improve therapeutic efficacy of bladder tumors. Our results show that blocking tumors of VEGF or/and EGFR pathways post PDT can effectively improve tumor response.

\section{Materials and Methods \\ Photosensitizer preparation}

A stock solution of hypericin (Molecular Probes Inc, USA) was prepared by adding $200 \mu \mathrm{l}$ of dimethyl sulfoxide, DMSO (Sigma Aldrich Inc, St Louis Mo, USA) to a $1 \mathrm{mg}$ vial of hypericin. The stock solution was further diluted in DMSO and PBS at a ratio of $1: 3 \mathrm{v} / \mathrm{v}$

*Corresponding author: Ramaswamy Bhuvaneswari, Division of Medical Sciences, National Cancer Centre Singapore, 11 Hospital Drive 169610, Singapore, E-mail: dmsram@nccs.com.sg

Received January 08, 2013; Accepted February 13, 2013; Published February 15,2013

Citation: Bhuvaneswari R, Thong P, Yuen GY, Olivo M, Chee SK (2013) Combined use of anti-VEGF and anti-EGFR Monoclonal Antibodies with Photodynamic Therapy Suppresses Tumor Growth in an In vivo Tumor Model. J Cancer Sci Ther 5: 100-105. doi:10.4172/1948-5956.1000192

Copyright: () 2013 Bhuvaneswari R, et al. This is an open-access article distributed under the terms of the Creative Commons Attribution License, which permits unrestricted use, distribution, and reproduction in any medium, provided the original author and source are credited. 
Citation: Bhuvaneswari R, Thong P, Yuen GY, Olivo M, Chee SK (2013) Combined use of anti-VEGF and anti-EGFR Monoclonal Antibodies with Photodynamic Therapy Suppresses Tumor Growth in an In vivo Tumor Model. J Cancer Sci Ther 5: 100-105. doi:10.4172/19485956.1000192

and injected intravenously into the tail vein based on the weight of the animal. At a dosage of $5 \mathrm{mg} / \mathrm{kg}$ approximately for a mouse weighing 25 g; $0.125 \mathrm{mg}$ of hypericin was administered.

\section{Murine xenograft tumor model}

MGH epithelial bladder cancer cell line was used to establish a murine xenograft model. The cells were grown in culture medium RPMI 1640 supplemented with $10 \%$ fetal bovine serum (FBS; Hyclone, UT, USA), IX L-Glutamine, 100 units/ml penicillin and streptomycin (Gibco, USA) and $1 \mathrm{X}$ sodium pyruvate in an atmosphere of $5 \% \mathrm{CO}_{2}$ at $37^{\circ} \mathrm{C}$. Once confluent the cells were washed with $1 \mathrm{X}$ phosphate buffered saline and trypsinized with $0.05 \%$ Trypsin-EDTA (Gibco, USA). 3 $\times 10^{6} \mathrm{MGH}$ bladder cancer cells were suspended in $150 \mu \mathrm{l}$ in Hank's balanced salt solution (Gibco, USA) and injected subcutaneously into the lower flanks of the mice. In 10-14 days the tumors grew to a size of approximately 6-7 mm. Male Balb/c nude mice, 6-8 weeks old, weighing an average of 24-25 g was purchased from Animal Resource Center, Western Australia.

\section{In vivo treatment protocol}

The mice were randomized into 6 groups (10 animals per group), (i) Control (mice with untreated tumors), (ii) PDT only, (iii) bevacizumab + cetuximab, (iv) PDT + bevacizumab, (v) PDT + cetuximab and (vi) PDT + bevacizumab + cetuximab. Treatment involved the intravenous injection of hypericin followed by irradiation with a light source consisting of filtered halogen light (Zeiss KL1500) fitted with a customized 560-640 $\mathrm{nm}$ band-pass filters. Light irradiation was performed $6 \mathrm{~h}$ post hypericin administration. A light dosage of $120 \mathrm{~J} / \mathrm{cm}^{2}$ and fluence rate $100 \mathrm{~mW} / \mathrm{cm}^{2}$ was used for PDT treatment. Bevacizumab and cetuximab were administered by intraperitoneal injections $(10 \mathrm{mg} / \mathrm{kg})$ at time 0 (immediately after light exposure), $24 \mathrm{~h}, 48 \mathrm{~h}$ and then every other day up to 90 days post PDT. For the combination therapy group of bevacizumab plus cetuximab, the inhibitors were administered alternately on a daily basis. Mice that were euthanized when either the tumor reached the $2 \mathrm{~cm}^{3}$ ethical limit or at the end of the 90-day monitoring period, the tumors were then harvested for analysis. Institutional Animal Care and Use Committee (IACUC), SingHealth, Singapore, approved all procedures and experiments were performed in accordance with international standards. Tumor volume was calculated by using the formula, volume $=(\pi / 6 \times \mathrm{d} 1 \times \mathrm{d} 2 \times \mathrm{d} 3)$, where $\mathrm{d} 1, \mathrm{~d} 2$ and $\mathrm{d} 3$ are tumor dimensions in 3 orthogonal directions. The percentage of tumor growth inhibition (TGI) was calculated by using the formula, TGI $=\left(\mathrm{V}_{\mathrm{c}}-\mathrm{V}_{\mathrm{e}}\right) / \mathrm{V}_{\mathrm{c}} \times 100 \%$, where $V_{c}$ is the average tumor volume in control groups and $V_{e}$ is the average tumor volume in the experimental groups.

\section{Immunoblotting}

Tissue lysate buffer (T-PER, Pierce, USA) along with protease inhibitor (Complete Mini, Roche, Germany) was added to the tumors that were crushed into powder in liquid nitrogen. Tissue and cell debris were removed by centrifugation and the lysate was stored at $-80^{\circ} \mathrm{C}$ until use for western blotting. Protein estimation of tumor lysates was performed using Biorad protein assay solution and was quantified using the GeneQuant pro machine (Biochrom, UK). Following the addition of sample buffer to the lysate, $50 \mu \mathrm{g}$ of protein was resolved onto the SDS gel and transferred to nitrocellulose membrane (Pall Corporation, USA). Membranes were blocked overnight with 5\% low fat milk powder-TBS-Tween and then washed thoroughly before probing with the primary antibody 1: 500 (VEGF and EGFR, Cell Signaling,
USA). After washing with TBS-Tween, the membranes were incubated with HRP-linked secondary antibody $(1: 1000)$ for $1 \mathrm{~h}$. The level of specific protein was visualized by chemiluminescence (Supersignal, Pierce Technology, USA). The membrane was then exposed to X-ray film (Hyperfilm ECL, Amersham Biosciences, UK) and the signal was detected using a film developer (Kodak M35, OMAT Processor, USA). The intensities of the signal were quantified using a densitometer (Syngene, USA) and analyzed with GeneTool (Syngene, USA).

\section{Evaluation of VEGF and EGFR using immunohistochemistry (IHC)}

Processing of the samples was done using tissue processor (Leica TP 1020, Germany). Briefly, the tissue samples were fixed in $10 \%$ of formalin for $24 \mathrm{~h}$, and then processed in an ascending series of ethanol and subsequently cleared with xylene and embedded in paraffin. The paraffin embedded samples of bladder tumors were cut at a thickness of $4 \mu \mathrm{m}$ using a microtome (Leica RM 2135, Germany). The sections were mounted on superfrost/plus slides (Fischer Scientific, USA) and airdried. On the day of staining, the slides were placed in $60^{\circ} \mathrm{C}$ oven for $1 \mathrm{~h}$ and immersed in xylene for $10 \mathrm{~min}$ before rehydrating in ethanol series. Sections were incubated with hydrogen peroxide for $10 \mathrm{~min}$ to block endogenous peroxidase activity. After which, the sections were incubated overnight at $4^{\circ} \mathrm{C}$ with primary antibodies, i.e., VEGF (Abcam, ab1316, 1:100) and EGFR (Abcam, ab2430-1, 1:50). To confirm the specificity of binding, normal mouse serum IgG1 (1:500) was used instead of primary antibody to serve as a negative control. Following extensive washing, sections were incubated for $30 \mathrm{~min}$ in the secondary biotinylated antibody, rinsed and followed by DAB Chromogen (Dako REAL EnVision Detection System, USA) for $10 \mathrm{~min}$. Sections were then counter-stained with Harris's hematoxylin and dehydrated in ascending grades of ethanol before clearing in xylene and mounting under a cover slip. Images were captured using imaging software (NIS Elements D, Nikon). The images were saved in TIFF format and NIH Image J (1.41o, W. Rasband, National Institute of Health, USA) software was used to analyze and quantify the expression of VEGF and EGFR. IHC scoring was performed based on the prevalence of VEGF and EGFR staining within the tumor (No staining $=0,<10 \%$ staining $=1$, $10-25 \%=2,26-50 \%=3,51-75 \%=4$ and $76-100 \%=5)$.

\section{Laser confocal endomicroscopy}

Confocal endomicroscopy was performed as follows: Fluorescein isothiocyanate (FITC) labeled dextran (MW $150 \mathrm{kDa}$, Sigma Aldrich, USA) solution was prepared at a concentration of $25 \mathrm{mg} / \mathrm{ml} .10 \mathrm{ml} \mathrm{FITC}$ dextran per gram weight of the animal was injected intravenously. The nude mice were then anesthetized with a cocktail of Hypnorm (0.315 $\mathrm{mg} / \mathrm{ml}$ fentanyl citrate and $10 \mathrm{mg} / \mathrm{ml}$ flanuisone, Janssen, NJ, USA) and Dormicum $(5 \mathrm{mg} / \mathrm{ml}$ midazolam $\mathrm{HCl}$, David Bull Laboratories, Australia). The skin overlaying the tumor was carefully removed to expose the tumor. In vivo fluorescence microscopic imaging of tumor blood vessels was carried out using a laser confocal endomicroscope, (Optiscan FIVE 1 system, Optiscan Pty Ltd., Victoria, Australia). A $488 \mathrm{~nm}$ laser provides excitation for in vivo fluorescence microscopy of tissue with a lateral resolution of $0.7 \mathrm{~mm}$ and an axial resolution of 7 $\mathrm{mm}$ within a field of view of $475 \mathrm{~mm} \times 475 \mathrm{~mm}$. The excitation light is coupled into a single optical fiber that acts as both a point source and a point detection pinhole for confocal imaging. A handheld rigid probe (model RBK6315A) with a probe shaft of length $150 \mathrm{~mm}$ and diameter $6.3 \mathrm{~mm}$ houses the miniaturized components of the $\mathrm{x}-\mathrm{y}$ scanning and $\mathrm{z}$-axis actuator. A footswitch allows the imaging depth to be controlled along the $\mathrm{z}$-axis range between surfaces to $250 \mathrm{~mm}$ subsurface in $4 \mathrm{~mm}$ 
Citation: Bhuvaneswari R, Thong P, Yuen GY, Olivo M, Chee SK (2013) Combined use of anti-VEGF and anti-EGFR Monoclonal Antibodies with Photodynamic Therapy Suppresses Tumor Growth in an In vivo Tumor Model. J Cancer Sci Ther 5: 100-105. doi:10.4172/19485956.1000192

steps, as well as to facilitate image capture. Serial 1024 pixels $\times 1024$ pixel images could be acquired at a frame rate of 0.7 frames/sec. For optimum image contrast, the laser power can be adjusted between 0 to $1000 \mathrm{~mW}$ at the $5.0 \mathrm{~mm}$ diameter distal tip of the probe in contact with the surface of the tissue. Fluorescence signals are collected via an emission filter with a bandwidth of $505 \mathrm{~nm}-750 \mathrm{~nm}$.

\section{Blood vessel analysis}

Blood vessel analysis was carried out using the Cell D imaging software (Olympus, Soft Imaging Solutions GmbH, Germany). The measurement function was used to measure the cross-sectional areas of the blood vessels visible within the field of view of the endomicroscope images by drawing user-defined regions of interest along the vessels. The mean vessel area for each animal group was calculated from images captured from three locations with high vessel density and that are 20 and $40 \mu \mathrm{m}$ below the surface of the tumor.

\section{Statistical analysis}

One-way analysis of variance (ANOVA) with the Bonferroni correction was performed to analyze the data obtained in this study using Prism 3.0 software (Graphpad Prism, San Diego, CA). A $p$ value of $<0.05$ was considered to be significant.

\section{Results}

\section{Assessment of tumor growth inhibition}

To investigate the long-term effectiveness of PDT and inhibitors, xenograft tumors were allowed to grow to sizes of 6-7 $\mathrm{mm}$ in diameter before PDT treatment was carried out and were measured three times a week for a 90-day period (Figure 1A). Tumors treated with the combination therapy of PDT + bevacizumab, PDT + cetuximab and the combination of PDT + bevacizumab + cetuximab exhibited significantly greater treatment response compared to control and PDT treated groups $(p<0.001)$. The difference in tumor response was significant between control and PDT only group $(p<0.05)$ and was highly significant between control and the combination therapy groups $(p<0.001)$. The treatment outcome in all the groups treated with PDT + one or more inhibitors was comparable. Next, we calculated the tumor growth inhibition (TGI) in different treatment groups against the growth of the control tumors (Figure 1B). 92\% TGI was observed in the groups treated with the combination therapy of PDT + bevacizumab + cetuximab, 90\% TGI was noticed in both PDT + bevacizumab and PDT + cetuximab treated tumors. In the tumors treated with the inhibitors bevacizumab and cetuximab, the TGI was $73.7 \%$. The PDT treated animals exhibited 57\% tumor growth inhibition. The tumors treated with combination therapy exhibited significantly greater TGI values compared to tumors treated with monotherapy. The treatment modalities in this study did not induce any signs of toxicity such as excessive weight loss, diarrhea or vomiting in the animals. No treatment-related death occurred.

\section{Assessment of VEGF and EGFR expression}

Immunohistochemistry was performed on paraffin embedded tissue for all the groups. As VEGF is a secreted protein, its expression was observed primarily in the cytoplasm and extracellular matrix. VEGF expression was found to be highest with intense staining in the PDT treated tumors (Figure 2 (b)) at 32\% (IHC score 3). In control tumors (Figure 2 (a)) and inhibitors (bevacizumab + cetuximab) treated tumors (Figure 2 (c)) around 14\% (IHC score 2) and $12 \%$
(IHC score 2) VEGF staining was observed, respectively. In the groups treated with PDT + bevacizumab (Figure $2(\mathrm{~d})$ ) and PDT + cetuximab (Figure 2 (e)), 4\% (IHC score 1) and 6\% (IHC score 1) staining was observed, respectively. Minimum VEGF expression of $1 \%$ (IHC score 1) was observed in the PDT + bevacizumab + cetuximab (Figure 2 (f)) combination therapy group.

EGFR expression was evaluated to investigate the anti-tumor activity in the various treatment groups. Tumors were harvested from the animals between 25-90 days, based on the maximum tumor volume limit or the completion of treatment. As EGFR is a membrane protein, staining of the cell membrane was observed. EGFR expression was found to be highest with increased staining in the PDT treated group (Figure 3 (b)) at 23\% (IHC score 2). In control tumors (Figure 3 (a)) and inhibitors (bevacizumab + cetuximab) treated tumors (Figure 3 (c)) around 14\% (IHC score 2) and 11\% (IHC score 2) EGFR staining was observed, respectively. In the groups treated with PDT + bevacizumab (Figure $3(\mathrm{~d})$ ) and PDT + cetuximab (Figure 3 (e)), 7\% (IHC score 1) and $4 \%$ (IHC score 1) staining was observed, respectively. Mild EGFR staining of $2 \%$ (IHC score 1 ) was observed in the PDT + bevacizumab + cetuximab (Figure 3 (f)) combination therapy group.

\section{Evaluation of different angiogenic proteins}

Expression of angiogenic proteins EGFR, VEGF, HER-2, STAT1,

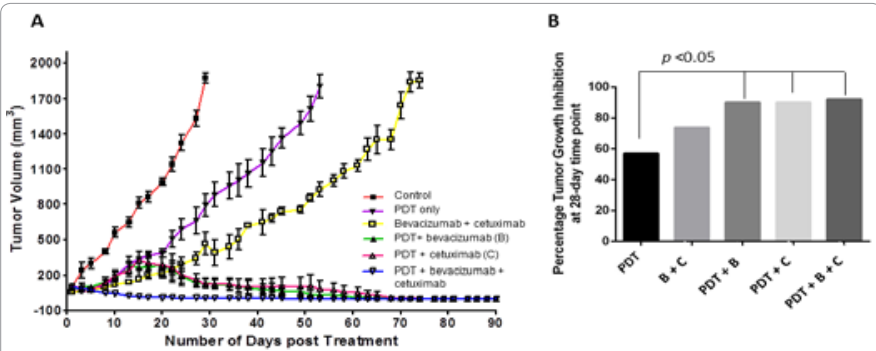

Figure 1: (A) Tumor volumes were charted against days to assess the tumor response in various treatment groups. The combination therapy groups of PDT + bevacizumab, PDT + cetuximab and PDT + bevacizumab + cetuximab exhibited greater tumor response in comparison with other groups. Each group represents the mean (error bars, SE) of 10 animals. (B) Percentage tumor growth inhibition was determined for each treatment group against control at 28 day time point by using the formula, tumor growth control $=\left(V_{c}-V_{e} / V_{c}\right) \times 100$, where $V_{c}$ is the average tumor volume in control groups and $V_{e}$ is the average tumor volume in the experimental groups.
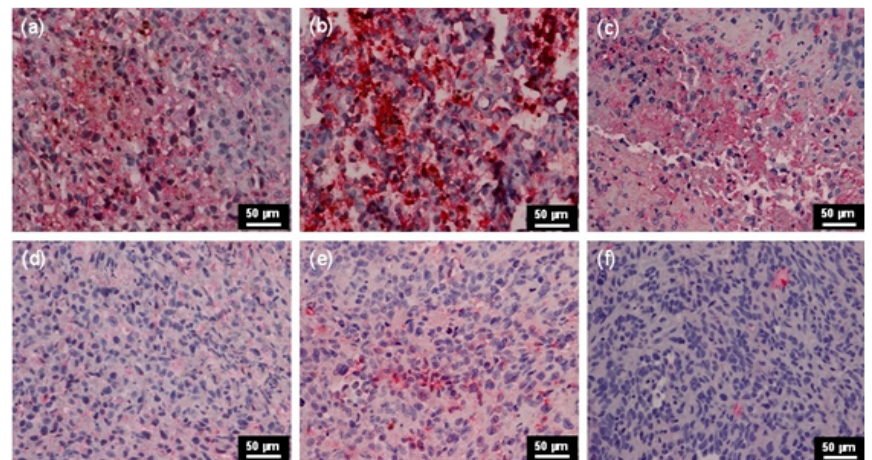

Figure 2: VEGF expression was assessed in tumors treated with various treatment regimens using immunohistochemistry (a) Control (untreated tumor), (b) PDT, (c) bevacizumab + cetuximab, (d) PDT + bevacizumab, (e) PDT + cetuximab and (f) PDT + bevacizumab + cetuximab. All sections are shown at a magnification of $\times 200$, scale bar $=50 \mu \mathrm{m}$. 
Citation: Bhuvaneswari R, Thong P, Yuen GY, Olivo M, Chee SK (2013) Combined use of anti-VEGF and anti-EGFR Monoclonal Antibodies with Photodynamic Therapy Suppresses Tumor Growth in an In vivo Tumor Model. J Cancer Sci Ther 5: 100-105. doi:10.4172/19485956.1000192

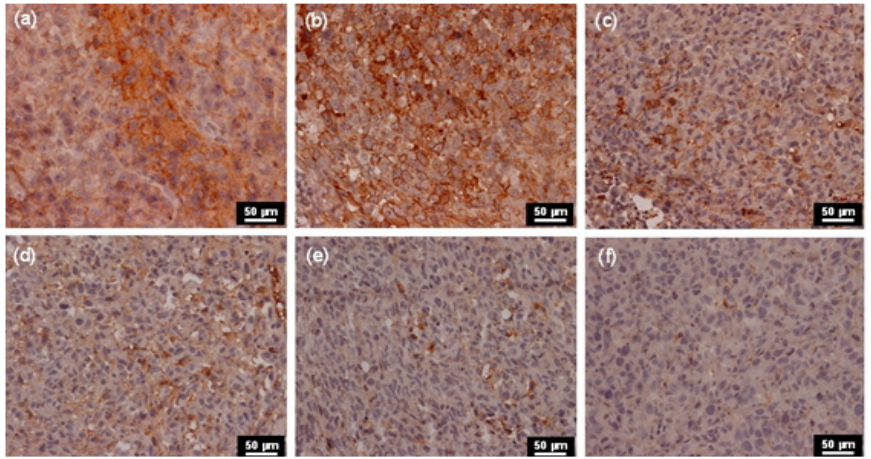

Figure 3: EGFR expression was assessed in tumors treated with various treatment regimens using immunohistochemistry (a) Control (untreated tumor), (b) PDT, (c) bevacizumab + cetuximab, (d) PDT + bevacizumab, (e) PDT + cetuximab and (f) PDT + bevacizumab + cetuximab. All sections are shown at a magnification of $\times 200$, scale bar $=50 \mu \mathrm{m}$.
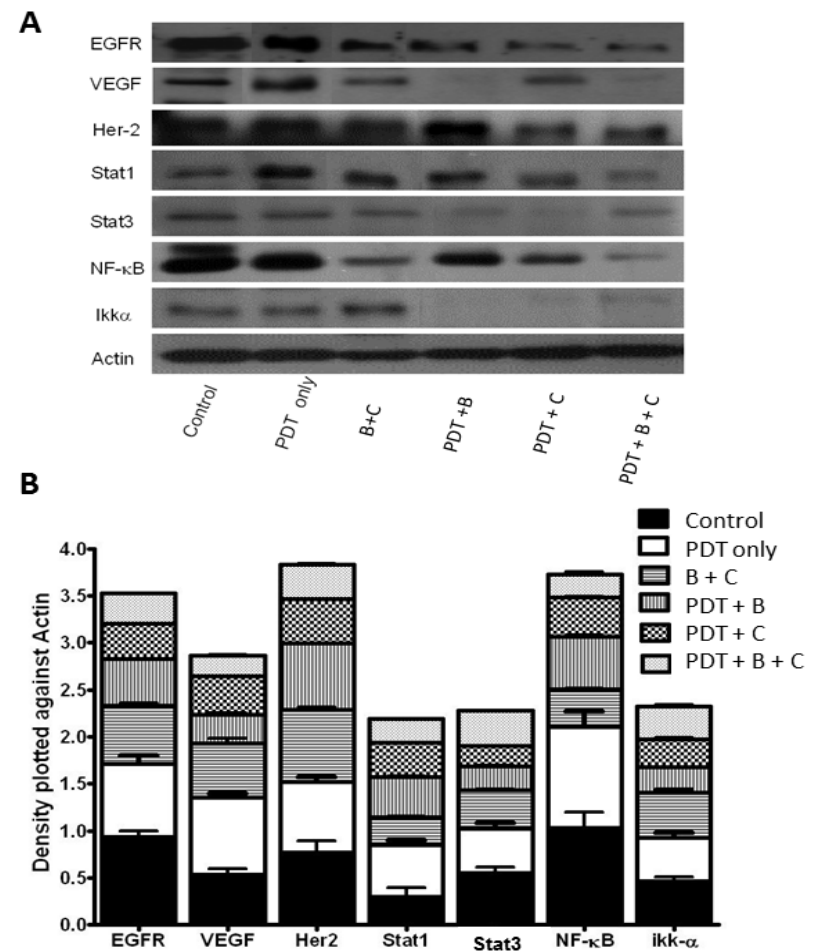

Figure 4: (A) Expression of EGFR, VEGF, Her-2, Stat1, Stat3, NF-kB, Ikka was detected in the treatment groups using western immunoblot analysis. Expression of actin was used to monitor protein loading, (B) Graph showing the intensity of bands plotted against Actin.

STAT3, NF- $\kappa$ B, and IKKa were detected in the treatment groups using western immunoblot analysis (Figure 4). EGFR and VEGF expression was downregulated in the PDT + inhibitors combination therapy groups. EGFR was highly suppressed in the PDT + bevacizumab + cetuximab treated tumors and VEGF was significantly downregulated in the PDT + bevacizumab and PDT + bevacizumab + cetuximab treated tumors. HER-2 (human epidermal growth factor receptor 2), also known as c-erbB2 and neu, belongs to the ErbB family of growth factor receptors with intrinsic tyrosine kinase activity. Downregulation of HER-2 was observed in the PDT + cetuximab and PDT + bevacizumab + cetuximab treated groups. The STAT protein family acts as a transcription factor in modulating pro- and anti-inflammatory responses and in this study PDT induced upregulation of STAT1 was observed. However, PDT in combination with angiogenic inhibitors attenuated this increase. STAT3 expression was downregulated to a greater extent in the PDT + bevacizumab and PDT + cetuximab treated tumors compared to PDT + bevacizumab + cetuximab. NF$\kappa \mathrm{B}$ activation is a general response to oxidative stress and can cause resistance to PDT treatment. Downregulation of NF- $\kappa B$ was noticed in the tumors treated with PDT + bevacizumab + cetuximab. IKKa is a serine kinase that is essential for NF- $\mathrm{KB}$ activation. IKKa expression was downregulated in all the groups treated with the combination of PDT and inhibitors.

\section{Visualization of tumor blood vessels}

Tumor blood vessels were visualized approximately $30 \mathrm{~min}$ after intravenous administration of FITC-dextran using confocal endomicroscopy. Control blood vessels (Figure 5A (a)) were well developed, dense and intact with an average vessel area (AVA) of around $12.5 \times 10^{4} \mu \mathrm{m}^{2}$ (Figure 5B) while those in treated groups (Figure 5A (c, $d, e, f)$ ) showed varying degrees of vessel damage following treatment. Tumors treated with PDT exhibited high vessel density (AVA: $12 \times$ $10^{4} \mu \mathrm{m}^{2}$ ) compared to other treatment groups (Figure 5A (b)). The PDT + bevacizumab (AVA: $4 \times 10^{4} \mu \mathrm{m}^{2}$ ), PDT + cetuximab (AVA: $7.5 \times 10^{4} \mu \mathrm{m}^{2}$ ) and PDT + bevacizumab + cetuximab (AVA: $2 \times 10^{4}$ $\mu \mathrm{m}^{2}$ ) combination treatment group showed the most post-treatment damage to shrunken vessels and leaky vessel walls resulting in a diffuse

A

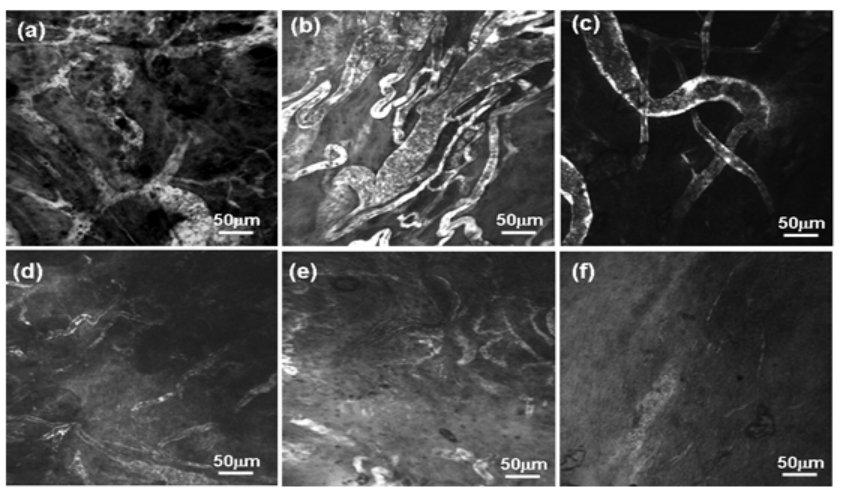

B

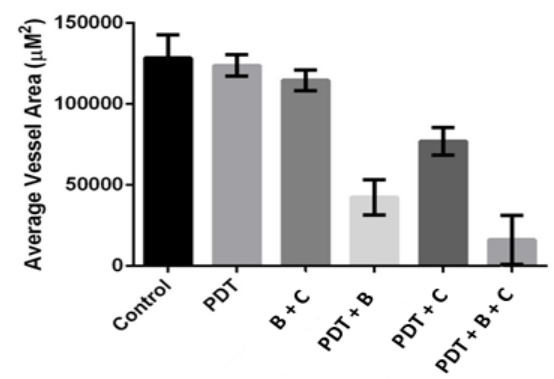

Figure 5: (A) Tumor blood vessels visualized approximately $30 \mathrm{~min}$ after intravenous administration of FITC-dextran in (a) control, (b) PDT only, (c) Bevacizumab + cetuximab, (d) PDT + bevacizumab, (e) PDT + cetuximab and (f) PDT + bevacizumab + cetuximab treated models using confocal endomicroscopy. Magnification $X 200$, scale bar $=50 \mu \mathrm{m}$. (B) Average vessel area for each animal group was calculated from images captured from three locations with high vessel density and that are 20 and $40 \mu \mathrm{m}$ below the surface of the tumor. 
Citation: Bhuvaneswari R, Thong P, Yuen GY, Olivo M, Chee SK (2013) Combined use of anti-VEGF and anti-EGFR Monoclonal Antibodies with Photodynamic Therapy Suppresses Tumor Growth in an In vivo Tumor Model. J Cancer Sci Ther 5: 100-105. doi:10.4172/19485956.1000192

fluorescence background. The vessel analysis results confirm with the treatment response observed in the different treatment groups.

\section{Discussion}

Photodynamic therapy is being successfully used for the treatment of early lung cancers [11] and early carcinomas of the oral cavity and larynx to preserve normal tissue and improve cure rates [12]. In the past 20 years PDT has been successfully used for the treatment of dermatological diseases, ophthalmic diseases, head and neck cancers, brain tumors, pulmonary and pleural mesothelial cancer, cardiovascular disease, gastroenterology cancer, urological disease and gynecological cancer [13]. However, since PDT is an oxygen consuming modality the hypoxic condition within the tumor tissue may induce the production of proangiogenic molecules, which can trigger recurrence by promoting angiogenesis. In this study we have attempted to suppress tumor growth post PDT by including anti-angiogenic agents to the protocol. Anti-angiogenic agents bevacizumab that is specific to VEGF and cetuximab that targets EGFR were administered after PDT to improve the overall tumor responsiveness in bladder tumors. The dosage and schedule for the administration of inhibitors was based on previous studies conducted on PDT and anti-angiogenesis $[14,15]$.

The tumor regression data demonstrated that combining bevacizumab + cetuximab with PDT can impede the angiogenic process and improve the response of treated tumors. The tumor volume of the PDT treated group was significantly greater than the bevacizumab treated and cetuximab treated groups as high fluence administered during treatment can deplete tumor oxygen to a large extent, releasing stress induced survival molecules that reduce the effectiveness of PDT and affect tumor control [16]. Although tumor regression was also observed in bevacizumab + cetuximab only treated group, complete cure was not observed. Thus targeting EGFR and VEGF without PDT treatment might not be sufficient to cause regression of most bulky tumors. One of the possible explanations for this observation may be related to pericytes that respond to angiogenic stimuli and promote endothelial stability through matrix deposition, and have macrophagelike function [17]. Also, the tumors overexpressing EGFR might not be sensitive to cetuximab. Although it is normally assumed that tumors overexpressing EGFR would respond well to anti-EGFR therapy, studies have demonstrated that the level of EGFR expression does not have enough impact on tumor response rates as a significant number of EGFR-positive tumors could be resistant to cetuximab $[18,19]$. Complete cure was noted in tumors treated with PDT and continued bevacizumab + cetuximab therapy. The data from the present study support the hypothesis that bevacizumab and cetuximab are capable of binding and neutralizing secreted VEGF and EGFR respectively, leading to regression of tumor vessels, normalizing surviving mature vasculature and preventing tumor recurrence post-PDT.

Expression of VEGF and EGFR that was observed using immunoblotting and IHC was suppressed in the tumors treated with PDT and inhibitors. The data in this study demonstrate that tumors treated with PDT express greater amounts of VEGF, which is consistent with an earlier report by Solban et al. [20] on subcurative PDT performed on an orthotopic model of prostate cancer that showed increased VEGF secretion. Also, significantly lower occurrence of VEGF was observed in the combination therapy of PDT and bevacizumab. On the other hand, previous studies have reported the downregulation of EGFR after PDT [21,22], in marked contrast the results of this study demonstrated an increase in EGFR expression post hypericin-mediated PDT. This observation could be attributed to numerous reasons such as the light/drug dosage, the complexity of tumor microenvironment and the properties of the photosensitizer [23]. Combined antitumor activity of bevacizumab and cetuximab with standard chemotherapy and radiotherapy is well documented in the treatment of different types of tumors and is reported to be more efficacious than individual monotherapies [24]. In this study, combination modality of PDT + bevacizumab + cetuximab was effective in reducing the expression of VEGF and EGFR which could have led to the greater tumor regression in this group.

The overexpression of HER2/neu is one of the important molecular markers of bladder cancer as it plays a major role in the aberrant proliferation of tumor cells. In this study, the tumors treated with cetuximab exhibited reduced expression of HER2. Similarly, a study on NCI-N87 cells has shown that cetuximab can completely inhibit EGFR-HER2 heterodimers, and also inhibit EGF-induced EGFR and HER2 phosphorylation [25]. EGFR has been shown to activate both STAT1 and STAT3 proteins. Ligand-induced recruitment of active Src kinases to the receptor complex appears to be a prerequisite for STAT phosphorylation [26]. STAT1 and STAT3 expression was attenuated when treated with EGFR inhibitor cetuximab, thus indicating that downregulation of EGFR can affect STAT1 and STAT3 phosphorylation. NF- $\mathrm{KB}$ is a survival-promoting factor and induces caspase suppression that prevents apoptosis. Therefore, reduction of NF-кB expression would be useful to increase the treatment efficiency. Reports have shown that EGFR can activate NF- $\kappa B$ through the phosphorylation of Iк-B $\alpha$ on serines $32 / 36$ thereby influencing the nuclear translocation of the p65 subunit. Ikka was downregulated to a greater extent in all the combination therapy groups compared to NF$\kappa \mathrm{B}$ expression that was downregulated only in the combination therapy of PDT + bevacizumab + cetuximab.

Laser confocal endomicroscopy offers in vivo surface and subsurface fluorescence imaging of tissue [27]. This technique has been successfully used for in vivo visualization of blood vessels and blood flow [28]. The goal of this experiment was to visualize changes in the morphology and functionality of tumor vasculature to evaluate the efficacy of the antiangiogenic therapy. The visualization of the blood vessels was performed either when the control tumors reached a maximum volume of $2 \mathrm{~cm}^{2}$ or at the end of the treatment. Blood vessels in control tumors were observed to be intact and dense, and the supply of nutrients by such effective networks of blood vessels could have enhanced the proliferation of control tumors. Angiogenesis was noticed in PDT only treated group. This could be due to the release of pro-angiogenic factors by hypoxic tumor cells as a response to PDTinduced stress. The results from this experiment revealed that the to fluence rate of $100 \mathrm{~mW} / \mathrm{cm}^{3}$ was high enough to induce oxidative stress and promote angiogenesis. Optimal results were obtained in the combination therapy group of PDT and inhibitors, in which the vessels were leaky and functional alterations, were also observed. Reduced microvessel density in bladder tumor has been reported previously, suggesting that the inhibition of VEGF, IL-8 and bFGF expression is responsible for the reduction in neovascularity observed in the tumors treated with MAb C225 [29]. Another study has shown that anti-EGFR C225 antibody exerts in vivo antitumor effect in androgenindependent prostate cancer in part by down-regulating the expression of IL-8, which in turn induces endothelial cell apoptosis leading to the regression of tumor neovascularity [30]. Based on our results, it can be hypothesized that PDT induced tumor damage can be sustained by the administration of bevacizumab and cetuximab thus inhibiting the production of angiogenic growth factors, most importantly VEGF and 
Citation: Bhuvaneswari R, Thong P, Yuen GY, Olivo M, Chee SK (2013) Combined use of anti-VEGF and anti-EGFR Monoclonal Antibodies with Photodynamic Therapy Suppresses Tumor Growth in an In vivo Tumor Model. J Cancer Sci Ther 5: 100-105. doi:10.4172/19485956.1000192

EGFR. Therefore, the combination of anti-angiogenic agents with PDT is a promising treatment modality.

In summary, the data from the present study supports the hypothesis that bevacizumab and cetuximab are capable of binding and neutralizing secreted VEGF and EGFR respectively, causing regression of tumor vessels, and preventing tumor recurrence.

\section{Acknowledgements}

The authors would like thank the National Medical Research Council (NMRC) Singapore for funding this study. We would also like to thank final year attachment students from Nanyang Technological University (NTU), Mr Ting Siong Luong and Mr Toh Zhi Qiang, for measuring the blood vessel areas from the captured images.

\section{References}

1. Jerjes W, Upile T, Hamdoon Z, Nhembe F, Bhandari R, et al. (2009) Ultrasoundguided photodynamic therapy for deep seated pathologies: prospective study. Lasers Surg Med 41: 612-621.

2. Chen B, Pogue BW, Hoopes PJ, Hasan T (2006) Vascular and cellular targeting for photodynamic therapy. Crit Rev Eukaryot Gene Expr 16: 279-305.

3. Dougherty TJ, Gomer CJ, Henderson BW, Jori G, Kessel D, et al. (1998) Photodynamic therapy. J Natl Cancer Inst 90: 889-905.

4. Gollinick SO, Evans SS, Baumann H, Owczarczak B, Maier P, et al. (2003) Role of cytokines in photodynamic therapy-induced local and systemic inflammation. Br J Cancer 88: 1772-1779.

5. Zetter BR (1998) Angiogenesis and tumor metastasis. Annu Rev Med 49: 407424.

6. Larsen AK, Ouaret D, El Ouadrani K, Petitprez A (2011) Targeting EGFR and VEGF(R) pathway cross-talk in tumor survival and angiogenesis. Pharmacol Ther 131: 80-90.

7. Shaheen RM, Ahmad SA, Liu W, Reinmuth N, Jung YD, et al. (2001) Inhibited growth of colon cancer carcinomatosis by antibodies to vascular endothelial and epidermal growth factor receptors. Br J Cancer 85: 584-589.

8. Yokoi K, Thaker PH, Yazici S, Rebhun RR, Nam DH, et al. (2005) Dua inhibition of epidermal growth factor receptor and vascular endothelial growth factor receptor phosphorylation by AEE788 reduces growth and metastasis of human colon carcinoma in an orthotopic nude mouse model. Cancer Res 65: 3716-3725.

9. Naumov GN, Nilsson MB, Cascone T, Briggs A, Straume O, et al. (2009) Combined vascular endothelial growth factor receptor and epidermal growth factor receptor (EGFR) blockade inhibits tumor growth in xenograft models of EGFR inhibitor resistance. Clin Cancer Res 15: 3484-3494.

10. Argiris A, Kotsakis AP, Hoang T, Worden FP, Savvides P, et al. (2013) Cetuximab and bevacizumab: preclinical data and phase II trial in recurrent or metastatic squamous cell carcinoma of the head and neck. Ann Oncol 24 220-225.

11. Moghissi K, Dixon K, Thorpe JA, Stringer M, Oxtoby C (2007) Photodynamic therapy (PDT) in early central lung cancer: a treatment option for patients ineligible for surgical resection. Thorax 62: 391-395.

12. Biel MA (2007) Photodynamic therapy treatment of early oral and laryngeal cancers. Photochem Photobiol 83: 1063-1068.

13. Huang $Z$ (2005) A review of progress in clinical photodynamic therapy. Techno Cancer Res Treat 4: 283-293.

14. Ferrario A, Gomer CJ (2006) Avastin enhances photodynamic therapy treatment of Kaposi's sarcoma in a mouse tumor model. J Environ Pathol Toxicol Oncol 25: 251-259.

15. Ferrario A, Fisher AM, Rucker N, Gomer CJ (2005) Celecoxib and NS-398 enhance photodynamic therapy by increasing in vitro apoptosis and decreasing in vivo inflammatory and angiogenic factors. Cancer Res 65: 9473-9478.
16. Gomer CJ, Ferrario A, Luna M, Rucker N, Wong S (2006) Photodynamic therapy: combined modality approaches targeting the tumor microenvironment Lasers Surg Med 38: 516-521.

17. Lu C, Kamat AA, Lin YG, Merritt WM, Landen CN, et al. (2007) Dual targeting of endothelial cells and pericytes in antivascular therapy for ovarian carcinoma. Clin Cancer Res 13: 4209-4217.

18. Vallbohmer D, Zhang W, Gordon M, Yang DY, Yun J, et al. (2005) Molecular determinants of cetuximab efficacy. J Clin Oncol 23: 3536-3544.

19. Ellis LM, Hoff PM (2004) Targeting the epidermal growth factor receptor: an important incremental step in the battle against colorectal cancer. J Clin Oncol 22: $1177-1179$.

20. Solban N, Selbo PK, Sinha AK, Chang SK, Hasan T (2006) Mechanistic investigation and implications of photodynamic therapy induction of vascular endothelial growth factor in prostate cancer. Cancer Res 66: 5633-5640.

21. Tsai T, Ji HT, Chiang PC, Chou RH, Chang WS, et al. (2009) ALA-PDT results in phenotypic changes and decreased cellular invasion in surviving cancer cells. Lasers Surg Med 41: 305-315.

22. Ahmad N, Kalka K, Mukhtar H (2001) In vitro and in vivo inhibition of epiderma growth factor receptor-tyrosine kinase pathway by photodynamic therapy Oncogene 20: 2314-2317.

23. Henderson BW, Gollnick SO, Snyder JW, Busch TM, Kousis PC, et al. (2004) Choice of oxygen-conserving treatment regimen determines the inflammatory response and outcome of photodynamic therapy of tumors. Cancer Res 64 2120-2126.

24. Press MF, Lenz HJ (2007) EGFR, HER2 and VEGF pathways: validated targets for cancer treatment. Drugs 67: 2045-2075.

25. Patel D, Bassi R, Hooper A, Prewett M, Hicklin DJ, et al. (2009) Anti-epiderma growth factor receptor monoclonal antibody cetuximab inhibits EGFR/HER-2 heterodimerization and activation. Int $\mathrm{J}$ Oncol 34: 25-32.

26. Olayioye MA, Beuvink I, Horsch K, Daly JM, Hynes NE (1999) ErbB receptorinduced activation of stat transcription factors is mediated by Src tyrosine kinases. J Biol Chem 274: 17209-17218.

27. Hoffman A, Goetz M, Vieth M, Galle PR, Neurath MF, et al. (2006). Confocal laser endomicroscopy: technical status and current indications. Endoscopy 38 1275-1283.

28. Goetz M, Thomas S, Heimann A, Delaney P, Schneider C, et al. (2008) Dynamic in vivo imaging of microvasculature and perfusion by miniaturized confocal laser microscopy. Eur Surg Res 41: 290-297.

29. Perrotte P, Matsumoto T, Inoue K, Kuniyasu H, Eve BY, et al. (1999) Antiepidermal growth factor receptor antibody C225 inhibits angiogenesis in human Transitional cell carcinoma growing orthotopically in nude mice. Clin Cancer Res 5: 257-264.

30. Karashima T, Sweeney P, Slaton JW, Kim SJ, Kedar D, et al. (2002) Inhibition of angiogenesis by the antiepidermal growth factor receptor antibody ImClone C225 in androgen-independent prostate cancer growing orthotopically in nude mice. Clin Cancer Res 8: 1253-1264. 